POPULATIONS

VUI.NEERABLES

\title{
Introduction. Les dimensions territoriales des vulnérabilités
}

Introduction. The territorial dimensions of vulnerabilities

\section{Maryse Gaimard}

\section{(2) OpenEdition}

1 Journals

Édition électronique

URL : https://journals.openedition.org/popvuln/991

DOI : $10.4000 /$ popvuln.991

ISSN : 2650-7684

Éditeur

LIR3S - Laboratoire Interdisciplinaire de Recherche "Sociétés Sensibilités Soin" (UMR 7366 CNRS-uB)

Édition imprimée

Date de publication : 1 mars 2019

Pagination : 9-17

ISBN : 978-2-918173-26-7

ISSN : 2269-0182

Référence électronique

Maryse Gaimard, «Introduction. Les dimensions territoriales des vulnérabilités », Populations vulnérables [En ligne], 5 | 2019, mis en ligne le 26 mai 2020, consulté le 28 février 2022. URL : http:// journals.openedition.org/popvuln/991; DOI : https://doi.org/10.4000/popvuln.991

Ce document a été généré automatiquement le 28 février 2022.

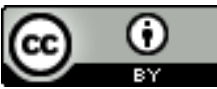

Les contenus de la revue Populations vulnérables sont mis à disposition selon les termes de la Licence Creative Commons Attribution 4.0 International 


\title{
Introduction. Les dimensions territoriales des vulnérabilités
}

\author{
Introduction. The territorial dimensions of vulnerabilities
}

Maryse Gaimard

1 Recouvrant de très nombreuses dimensions, qu'elles soient sanitaires, démographiques, économiques, géographiques, politiques, psychologiques, philosophiques ou encore sociologiques, la vulnérabilité est devenue l'objet de très nombreuses recherches pluri et inter-disciplinaires à l'échelle internationale (Thomas, 2008). Dans une société moderne souvent considérée comme une "société du risque» (Beck, 2001), la vulnérabilité touche des individus ou des groupes sociaux soumis à un contexte extraordinaire marqué par une situation de risques prononcés par rapport à une situation de référence (politique, environnementale, géographique, historique, etc.) qui est, elle, caractérisée par la normalité. En d'autres termes, elle qualifie l'état de personnes ou de groupes exposés à des risques qui se déploient souvent selon plusieurs dimensions synchroniques (par exemple, les risques de maladie, de pauvreté et d'isolement sont souvent liés) et dont la nature peut varier. Il y aurait donc dans certaines situations un faisceau de facteurs combinés conduisant un individu ou une population à se vulnérabiliser ou à se penser comme tel.

2 Ainsi, la vulnérabilité peut rendre compte de l'ensemble des facteurs qui constituent la fragilité d'une société, d'un territoire, d'une population, d'une structure, d'un écosystème, d'une organisation ou encore d'un système politique. Elle peut également faire référence à des disparités sociales (pauvreté, exclusion, relégation spatiale, etc.), à des catégories évolutives d'âge ou de santé (vieillesse, maladie, handicap, etc.), à des déséquilibres conjoncturels ou systémiques (économiques, environnementaux, psychologiques), etc. Partant de là, la vulnérabilité est nécessairement relative, donc à situer ou, dit autrement, à contextualiser tant ses explications sont pluricausales.

3 La notion de vulnérabilité présente ou contient en elle-même des enjeux éthiques et politiques majeurs: l'identification d'individus ou de populations en situation de vulnérabilité conduit à reconnaître leurs difficultés, mais peut également participer à leur stigmatisation (Goffman, 1975). Elle s'inscrit en outre dans la durée et selon des 
étapes plus ou moins marquées qui constituent un processus de vulnérabilisation des populations que le chercheur doit mettre au jour.

Dans ce champ de recherche, la dimension spatiale et territoriale des différentes formes de vulnérabilité revêt une importance capitale par les interactions qui s'effectuent entre les populations en situation de vulnérabilité et le territoire qu'elles occupent. Les vulnérabilités (et la vulnérabilisation) des populations se différencient selon le territoire habité, ce qui entraîne des disparités, voire des inégalités, entre individus. Ainsi, certains territoires, de par leur contexte démographique (vieillissement de la population), géographique et écologique (climat, conditions environnementales, salubrité, etc.), économique (déprise industrielle), social, culturel, politique (conflits) produisent des situations de fragilités pour les populations (Gaimard et al., 2018).

Dans les articles qui composent ce numéro de Populations vulnérables, l'approche territoriale est une entrée privilégiée : territoires socialement et spatialement relégués (espaces périurbains, espaces ruraux, bidonvilles) qui accueillent des populations vulnérables, en exclusion sociale et économique. Ces territoires peuvent renforcer la vulnérabilité, voire la logique de l'exclusion pour les populations les plus fragiles. Les espaces urbains sont aussi vecteurs de vulnérabilité par la densité de population car la ville dense favorise l'anonymat et l'isolement. Les différents auteurs sont ainsi amenés à discuter des dimensions territoriales de la vulnérabilité.

6 Le territoire est, comme le concept de vulnérabilité, une notion au cœur des débats scientifiques mais aussi politiques et économiques. La définition du territoire est complexe et cette notion remplace celle d'espace dès les années 1960, années pendant lesquelles l'aménagement du territoire s'est développé avec la prise en compte des besoins locaux. Le territoire, qui peut de prime abord être considéré comme un concept géographique, a été approché par différentes disciplines qui lui donnent des acceptions diverses. Un territoire se présente comme un système, un espace organisé où interagissent les différents acteurs qui le composent et le font vivre. Selon Guy Di Méo (1998), le territoire, souvent abstrait, vécu et ressenti plus que visuellement repéré, est un espace social et un espace vécu. «Le territoire témoigne d'une appropriation à la fois économique, idéologique et politique (sociale donc) de l'espace par des groupes qui se donnent une représentation particulière d'eux-mêmes, de leur histoire, de leur singularité, etc.» Pour Kourtessis-Philippakis (2011) «Le territoire est un investissement affectif et culturel que les sociétés placent dans leur espace de vie. Le territoire s'apprend, se défend, s'invente et se réinvente. Il est lieu d'enracinement... Un territoire est un lieu de vie, de pensée et d'action dans lequel et grâce auquel un individu ou un groupe se reconnaît, dote ce qui l'entoure de sens et se dote lui-même de sens, met en route un processus identificatoire et identitaire. » Il est généralement admis que le territoire est une construction sociale « qui se traduit, soit par un contrôle territorial, soit par un aménagement ou une structuration de l'espace.» Il apparait comme un espace identifié caractérisé par des pratiques et des représentations.

7 Les populations vivent le territoire, l'influencent et ce dernier agit aussi sur les individus. Du jeu des différents acteurs, "il en résulte des territoires en "tension", c'est-à-dire dont l'équilibre dynamique repose sur un ensemble d'interrelations qui ne cessent de se modifier dans le temps. Elles sont en effet fondées sur des contraintes qui ne sont jamais complètement prévisibles, compte tenu de l'infinité de phénomènes qui se déroulent simultanément, en obéissant à des temporalités différentes, et modifient 
sans cesse le contexte décisionnel des acteurs.» (Moine, 2006). Le concept de vulnérabilité territoriale intègre les interactions entre la société et l'environnement physique pour un contexte géographique particulier. L'endroit géographique (zone urbaine, région, territoire) constitue un élément de la vulnérabilité des populations, dans lequel les composantes biophysiques, sociales et culturelles sont indissociables.

8 Ainsi le territoire peut créer, entretenir, exacerber des vulnérabilités ou au contraire les atténuer voire les rendre plus supportables. Au-delà des conditions naturelles, physiques, climatiques et écologiques du territoire, ses caractéristiques économiques, sociales ou culturelles peuvent rendre certaines populations vulnérables. Les interactions entre les vulnérabilités et les territoires sont donc multiples. En effet, certaines formes de vulnérabilité sont spécifiques à des espaces géographiques donnés.

La revue Populations vulnérables, dans la suite de ce numéro, propose une réflexion sur les dimensions territoriales des vulnérabilités à partir de travaux novateurs faisant la part belle à l'interdisciplinarité autant qu'à une perspective transnationale, seule à même de relayer la diversité et l'hétérogénéité évoquées précédemment. Sept articles abordent les interactions entre la vulnérabilité des populations et le territoire qu'elles habitent.

Dans son article Conditions sociales, registres de vulnérabilité et territoires de vie dans la France d'aujourd'hui, Hervé Marchal revient en détail sur le concept de vulnérabilité, son évolution au cours des années, ses différentes approches disciplinaires, avant d'analyser les liens entre des formes de vulnérabilité et des territoires précis. L'auteur porte un regard de sociologue sur les dimensions territoriales des vulnérabilités. Il s'appuie, pour cela, sur ses travaux de recherche empiriques menés dans des zones résidentielles du périurbain éloigné d'une métropole régionale, dans une cité d'habitat social et dans un bidonville. Il discerne ainsi plusieurs formes de vulnérabilités rencontrées dans la société française et identifie «différents registres de vulnérabilité » qui selon lui «en disent long sur l'existence de fortes disparités non seulement spatiales mais aussi sociales ». En outre, l'auteur intègre les différentes formes de vulnérabilités qu'il analyse dans ses divers terrains dans une approche idéaltypique des conditions sociales. Il distingue ainsi la condition intro-sociale à travers l'étude des zones périurbaines, la condition hétéro-sociale observée dans un habitat social déshérité et la condition extro-sociale à partir d'une recherche dans un bidonville.

11 Jean-François Léger, Décroître pour rebondir: le déclassement des villes moyennes, une opportunité pour repenser la ville, apporte le point de vue du démographe sur les fragilités de certaines zones urbaines par le prisme des villes moyennes. Pour l'auteur, les villes moyennes souffrent du mouvement de métropolisation de ces dernières décennies, favorisant l'expansion des grandes agglomérations au détriment des unités urbaines plus petites. Après avoir précisé ce qu'il entend par ville moyenne, l'auteur présente les principales caractéristiques de l'évolution de ces villes et les différentes étapes qui ont conduit à la dynamique urbaine actuelle. Au-delà de l'expérience de la crise et de la vulnérabilité des populations y résidant, les villes moyennes montrent un certain nombre d'atouts qui pourraient assurer leur renouveau. L'auteur donne une dimension comparative à son analyse selon la proximité, ou non, des villes moyennes d'une grande agglomération.

12 Les trois articles suivants envisagent la vulnérabilité sociale et économique des populations en lien avec leur territoire de rattachement. Sandrine Gombert- 
Courvoisier et al. situent l'analyse de populations vulnérables dans le contexte de la transition écologique. Leur article Vulnérabilité des ménages et sentiment de déclassement social : l'adoption de comportements écologiques peut-elle contribuer à satisfaire les motivations identitaires dans un contexte territorialisé ? fait suite à une présentation de leurs travaux lors des $27^{e}$ journées de la Société d'écologie humaine (SEH) intitulées «Vulnérabilités et territoires", organisées à l'université de Bourgogne (Dijon) en octobre 2016. Les auteurs s'interrogent sur la possibilité que l'adoption de comportements écologiques par les ménages leur permette de surmonter le sentiment de déclassement social qu'ils ressentent suite à l'augmentation des inégalités et la détérioration du pouvoir d'achat ». Pour cela, ils définissent et identifient les perturbateurs de la qualité de vie et les comportements écologiques. L'analyse repose sur une méthodologie associant l'exploitation des bases de données de l'INSEE et une enquête par questionnaire dans trois territoires de la Gironde : la métropole de Bordeaux, le territoire périurbain du Créonnais et le territoire rural du Pays Foyen. Les résultats laissent apparaître des différences territoriales qu'il importe de prendre en compte.

La vulnérabilité des populations est abordée sous l'angle des vulnérabilités résidentielles par Jean-Baptiste Daubeuf. L'expérience plurielle de la vulnérabilité au sein des bidonvilles qu'il propose fait état des stratégies déployées par les habitants d'un bidonville pour survivre. À travers un travail ethnographique très fin et approfondi d'un bidonville occupé par des migrants Roms, l'auteur interroge les multiples facettes que peut prendre la vulnérabilité dans cette forme d'habitat. Il emmène le lecteur dans les différentes étapes de la migration de ces personnes, de leur village roumain jusqu'à leur installation dans le bidonville, en passant par leurs différents points de chute. Les fragilités sont économiques, monétaires mais proviennent aussi des constructions dans lesquelles vivent les personnes ainsi que dans les rapports difficiles qu'ils entretiennent avec les institutions et la société française. Ces fragilités, qui se combinent entre elles de manière complexe, s'expriment et touchent différemment les habitants et leur famille. Dans ce contexte difficile, les individus bénéficient de la protection apportée par le collectif.

14 La désindustrialisation et la fermeture des usines sont des facteurs de vulnérabilité pour les travailleurs et leur famille. Thomas Venet dans son article $L a$ désindustrialisation comme vecteur de vulnérabilité territoriale, analyse ces phénomènes en Picardie, une des régions les plus touchées par la crise de l'industrie. Il montre les bouleversements qui ont affecté ce territoire et conduit à fragiliser une partie de la population en analysant «conjointement des dynamismes sociaux, économiques et démographiques situés à des échelles géographiques fines ». L'approche privilégiée par l'auteur est géographique et démographique, par les modifications de la structure des emplois et l'évolution du chômage. Ces facteurs de vulnérabilisation s'expriment différemment selon les communes, amenant ainsi l'auteur à localiser les phénomènes par des analyses statistiques à un échelon géographique très fin et à proposer une typologie des territoires picards désindustrialisés.

15 Deux articles proposent une analyse de la vulnérabilité des populations liée au territoire qu'elles occupent par la question de la santé. Andry Herisoa Andrianasolo et al., Dimensions de la vulnérabilité liée au paludisme dans deux zones de Madagascar : apports d'une approche mixte, présentent une analyse comparative de la vulnérabilité au paludisme à Madagascar ${ }^{1}$. Le risque de paludisme et sa transmission dépendent en effet grandement du contexte géographique, climatique et socio-économique du territoire. 
Les auteurs analysent les déterminants de la vulnérabilité associée au paludisme dans deux régions, Ankazobe (Hautes Terres) et Brickaville (côte est), qui présentent des contextes environnementaux bien différents. Elle s'attache à montrer pourquoi, malgré les diverses campagnes et programmes de lutte contre le paludisme, sa prévalence demeure élevée, ne baissant que très lentement. Pour sa démonstration, l'auteure s'appuie sur une approche mixte combinant une enquête quantitative par questionnaire auprès de 4000 personnes et une enquête qualitative regroupant une soixantaine d'entretiens.

Katia Mazalovic et al., dans un article intitulé Le repérage des patients en situation de précarité par les médecins généralistes, abordent la vulnérabilité des populations par le prisme des inégalités de santé. Les inégalités sociales et économiques conduisent un certain nombre de personnes à vivre dans une certaine précarité, précarité qui se manifeste également face à l'accès aux soins, les rendant vulnérables sur le plan sanitaire. Des efforts spécifiques de prise en charge de ces personnes sont nécessaires. or, pour cela, il est nécessaire d'identifier cette population vulnérable. Cette identification peut être réalisée par le médecin généraliste lors des consultations, en mesurant la précarité par un score, le score EPICES (Évaluation de la précarité et des inégalités de santé dans les centres d'examens de santé). À partir d'une enquête réalisée en milieu rural auprès de médecins généralistes et de leurs patients, l'auteure analyse les écarts du repérage de la précarité entre l'évaluation spontanée des médecins et le score EPICES. Elle met en évidence les facteurs de concordances ou de discordance entre ces deux méthodes d'identification des personnes précaires et vulnérables.

17 À travers une diversité d'approches, de terrains, de méthodologies, les articles présentés dans ce dossier s'attachent à analyser les interactions entre le territoire et les populations qui les habitent comme des vecteurs de vulnérabilités.

\section{BIBLIOGRAPHIE}

Beck U. (2001), La société du risque, Paris, Aubier.

Di Méo G. (1998), Géographie sociale et territoire, Paris, Nathan.

Gaimard M., Gateau M. et Ribeyre F. (2018), Vulnérabilités et territoires, Nancy, Kaïros, collection "Questions humaines».

Goffman E. (1975), Stigmate. Les usages sociaux du handicap, Paris, Éditions de Minuit, [1963].

Kourtessis-Philippakis G. (2011), « La notion de territoire : définitions et approches », in Kourtessis-Philippakis G. et Treuil R. (dir.) Archéologie du territoire, de l'Égée au Sahara, Cahiers archéologiques de Paris 1, Paris, La Sorbonne, p. 7-13.

Moine A. (2006), « Le territoire comme un système complexe : un concept opératoire pour l'aménagement et la géographie ", L’Espace géographique, 2006, t. 35, nº 2, p. 115-132. 
Thomas H. (2008), Vulnérabilité, fragilité, précarité, résilience, etc. De l'usage et de la traduction de notions éponges en sciences de l'homme et de la vie, Recueil Alexandries $\mathrm{n}^{\circ} 13$, disponible sur : http:// www.reseau-terra.eu/article697.html.

\section{NOTES}

1. Cet article a fait l'objet d'une communication aux $27^{e}$ journées de la Société d'écologie humaine (SEH) intitulées « Vulnérabilités et territoires », organisées à l'université de Bourgogne (Dijon) en octobre 2016.

\section{INDEX}

Mots-clés : vulnérabilité, territoires

Keywords : vulnerability, territories

\section{AUTEUR}

\section{MARYSE GAIMARD}

Professeure de démographie

Université de Bourgogne-Franche-Comté, LIR3S-UMR 7366

maryse.gaimard@u-bourgogne.fr 